\title{
Current status of immunotherapy in acute myeloid leukemia
}

\author{
Wenrui $\mathrm{Li}^{1 \text {,** }}$ \\ ${ }^{1}$ Department of Pathophysiology, Key Laboratory of Cell Differentiation and Apoptosis, Shanghai Jiao Tong University School of \\ Medicine, Shanghai, China.
}

\begin{abstract}
Acute myeloid leukemia (AML) is a rapidly progressive, poor prognosis malignant tumor caused by hematopoietic stem cells/progenitor cells. In recent years, there have been significant advances in basic and preclinical research on AML. Compared with traditional chemotherapy, hematopoietic stem cell transplantation (HSCT) significantly improved prognosis. However, with high recurrence rates and low 5-year survival rates, more and more attention has been focused on immunotherapy strategies for AML. Given the immunological characteristics of AML and the mechanisms of immune escape, ongoing efforts are aimed at improving the strategy of immunotherapy and the design of novel therapies, such as vaccines, monoclonal antibodies, chimeric receptor-engineered $\mathrm{T}$ cells (CAR-T), and checkpoint inhibitors, which hopefully can deliver higher specificity and efficacy in AML therapy. In this review, we provide an overview of the immunological characteristics of conventional AML therapies, explore immune avoidance mechanisms, and describe the mechanisms of active and passive immunotherapies and current clinical trials.
\end{abstract}

\section{Introduction}

Acute myeloid leukemia (AML) is a fast-growing malignant blood cancer caused by the overproliferation of progenitor cells at different stages of myeloid primitive cell development. In AML, primitive myeloid cells and immature myeloid cells in bone marrow and peripheral blood go through abnormal proliferation, leads to rapid growth of a large number of abnormal cells in bone marrow and blood, which interferes with normal hematopoietic function of the human body. They prevent the marrow from making normal red blood cells, white blood cells and platelets. With fewer healthy blood cells, the body can't fight infections or stop bleeding. The clinical manifestations include anemia, dyspnea, abnormal metabolism, blood stasis, hemorrhage, infection, fever, etc. The exact cause of acute myeloid leukemia remains unclear, but is associated with the environment, ionizing radiation, chemical exposure, use of alcohol and tobacco, and specific responses to certain viral infections. In addition, recent studies on the frequency of gene mutations and some susceptible biomarkers have found that AML may be the result of genetic and environmental factors.

\section{Traditional treatments for AML}

\subsection{Hematopoietic stem cell transplantation (HSCT) for AML}

Bone marrow or blood hematopoietic stem cell transplantation (HSCT) is a traditional treatment for

*Corresponding author: 1wr_01@sjtu.edu.cn patients who have AML. It replaces the unhealthy blood-forming cells (stem cells) with healthy ones. After achieving a first complete chemotherapy-induced remission (CR1) in AML, a postremission therapy is necessary to prevent disease relapse. Myeloablative conditioning followed by allogeneic stem cell transplantation (alloSCT) is the earliest immunotherapy modality that is still widely used and is an effective treatment option for young AML patients in CR1.[1] Donor's healthy natural killer cells and $\mathrm{T}$ lymphocytes recognize and target the malignant cells and replace them. For some people, transplant can cure their disease.

\subsection{Immune system against AML}

GVL is a graft-versus-leukemia effect closely associated with allogeneic hematopoietic progenitor cells and stem cell transplantation. The GVL effect is the underlying reason why stem cell allografts can cure hematologic malignancies [2]. The natural killer cells and $T$ lymphocytes can recognize and target the recipient's malignant cells. It was found that the rate of leukemia recurrence in patients with acute or chronic graft-versus-host disease (GVHD) was 2.5 times higher than in patients without GVHD. In addition, the recurrence rate of leukemia was higher in patients with the syngeneic bone marrow transplantation than in patients with the allogeneic bone marrow transplantation. Therefore, it is speculated that the donor lymphocytes may have a certain degree of attacking effect on the leukemia cells in the recipient, which is called the GVL effect. 


\subsection{Immune escape in AML}

Studies show that both the innate and adaptive immune systems can recognize AML cells, eliminate leukemia cells or maintain a balance to prevent tumor growth.[3] AML cells are impressionable to T cells and NK cells attack by expressing co-stimulating molecules and ligands of leukemia-associated antigens, MHC and NK cell-activated receptors.[4] Natural killer (NK) cells, the backbone of the innate immune system, support the development of an effective adaptive anti-tumor immune response by secreting cytotoxic and cytokines that exert direct anti-tumor effects and indirectly contribute to tumor control through communication with other immune cells.[5]

Natural killer cells, T cells, and dendritic cells (DC) play an important role in the immune monitoring of acute myeloid leukemia, so their anti-leukemia properties have been applied to different immunotherapy strategies. Although NK cells can target cancer cells and have been shown to be useful in AML immunotherapy, some hematologic malignancies, including AML, can cause NK cell function to significantly impaired. Thus, AML can escape the immune surveillance of NK cells through a series of mechanisms that contribute to the dysfunction of the immune response, leading to immune escape.[5]

In this context, clinical trials have explored multiple vaccines, cytokines, and therapies to enhance T-cell immunity, improve target cell sensitivity, and strategies to directly attack AML cells to overcome immune escape.[6] Despite advances in AML treatment in recent decades, survival rates have not improved significantly due to the limitations of treatment and high incidence of leukemia recurrence, suggesting the need for new treatments.[7, 8]
In addition to the emerging field of chemotherapy and targeted therapies, immunotherapy for AML is an important and attractive area of research.

\section{Immunotherapy for AML}

\subsection{Active immunotherapy}

Active tumor immunotherapy is to inject the tumor antigen and let it induces the innate immune effector cells, which identify the specific tumor antigens, mediate specific immune response and secrete antibodies. The most commonly used are vaccines. Tumor vaccines Researchers have been worked on the tumor vaccines for long and some types of vaccines are used in AML treatment. Most clinical trials have shown effects and clinical benefit in AML, such as peptide vaccines $[9,10]$, DNA vaccines[11] and dendritic cell-based vaccines[12, 13].

\subsubsection{Autologous whole AML cell vaccines}

Tumor cell vaccines use modified autologous whole tumor cells to stimulate the organism to produce an anti-tumor response. Considering the AML cells surface contains lots of known and unknown antigens, autologous whole AML cells seem to help stimulate AML specific immune responses against multiple antigens, providing therapeutic vaccines for specific individuals. This kind of vaccines can effectively reduce the probability of immune escape. Studies have been done on trials using autologous whole AML cells, revealing the immune response was increased as the result of autoimmunization. [14]

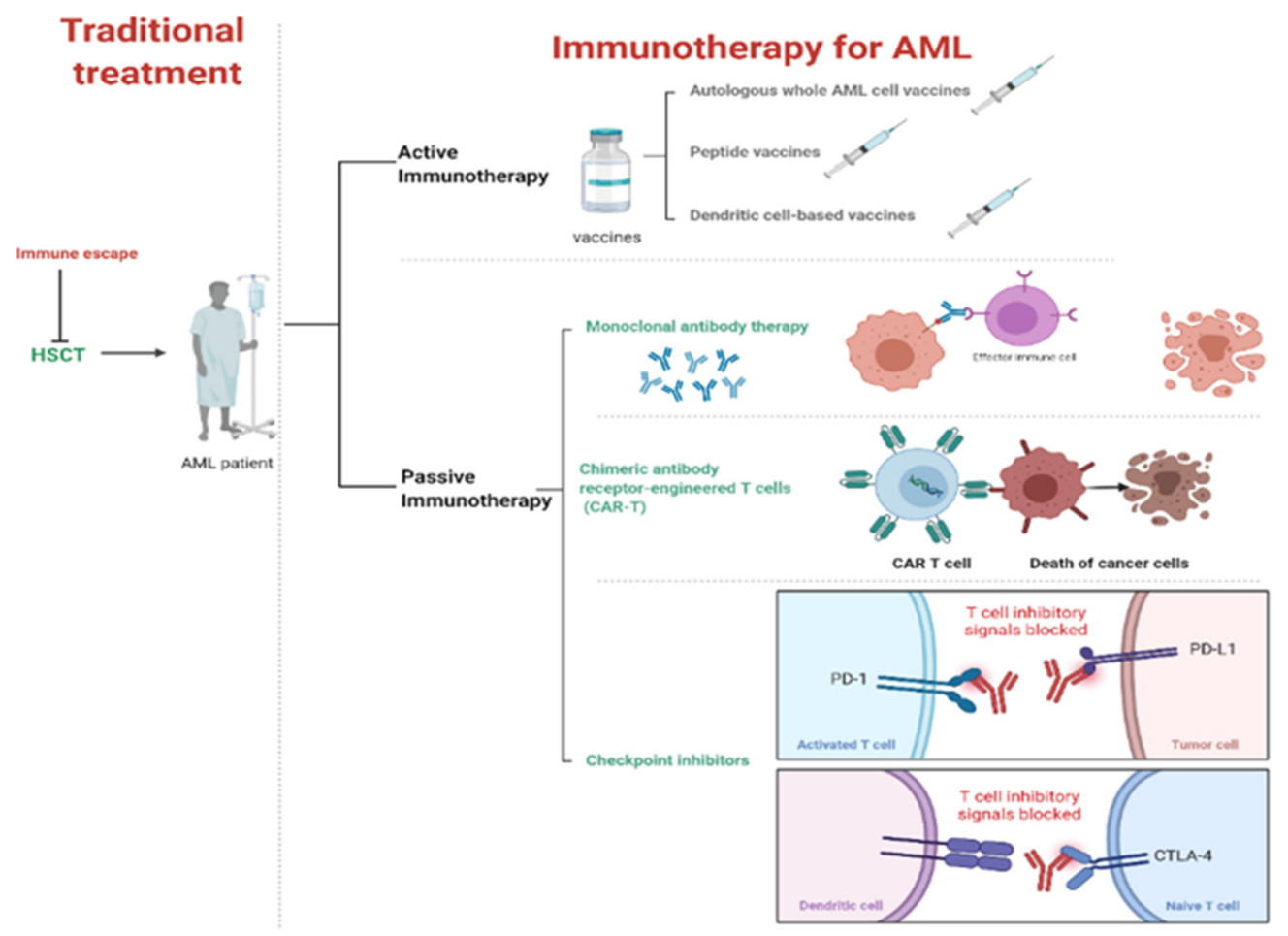

Fig. 1. Immunotherapy for AML. Created with BioRender.com 


\subsubsection{Peptide vaccines}

Peptide vaccines are vaccines that induce immune responses prepared by chemical peptide synthesis techniques based on the amino acid sequences of certain epitopes known or predicted from leukemia-associated antigens (LAAs).[15]Peptide vaccines are often synthetic vaccines and resemble naturally occurring proteins from pathogens. Peptides from tumor associated antigens are used to induce effective anti-tumor T cell response.

Peptide vaccines developed for AML have been used in clinical trials against different leukemia-associated antigens, such as protease-3-derived epitope peptide (PR1), Wilms tumor 1 (WT1) antigen, hyaluronic acid-mediated motor receptor (RHAMM), and melanoma preferentially expressed antigen (PRAME).[16]

\subsubsection{Dendritic cell-based vaccines}

Dendritic cells (DCs) are cells that play a role in antigen presentation in the mammalian immune system. Peripheral dendritic cells capture and process antigens, express lymphocyte co-stimulatory molecules, migrate to lymphoid organs, and secrete cytokines to initiate immune responses.[17] They act as messengers between the innate and the adaptive immune systems. Dendritic cells can now be readily obtained and allowed to be used in molecular and cell biological analysis. Thus, DC-based vaccines have attracted much attention in recent years with the realization that these cells are a powerful tool for manipulating the immune system.

Dendritic cell-based vaccine is a hopeful strategy for the elimination of disease in patients with acute myeloid leukemia (AML), especially for these with high relapse risk and not suitable for hematopoietic stem cell transplantation. Once injected the DC vaccines, DCs can be produced in 3 days and combined with Toll-like receptor signaling-induced cell maturation. These mature DCs bind to RNA encoding the leukemia-associated antigen Wilm's tumor protein 1 and secrete the antigen in melanoma to stimulate an immune response based on AML-specific T cells. $[12,13]$. Studies have shown that these improved DCs have strengthened capacity for the induction of tumor-specific immune responses in vitro and in vivo.[18-20]

\subsection{Passive immunotherapy for AML}

Passive immunity is a method by which the body acquires specific immunity by passively receiving antibodies or sensitized lymphocytes. The passive immunotherapy approach is to give patients donated or laboratory-made components of their immune system to help them combat the disease. Passive immunotherapy using antibodies is frequently used in cancer treatment.[21]. Its effects are rapid and has no latency compare to active immunotherapy. Once the antibodies or sensitized lymphocytes enter the body, the patient acquires immunity immediately. To date, several passive immunotherapy strategies have been studied, including adoptive immunotherapy, chimeric antibody receptor engineered T cells (CAR-T), and checkpoint inhibitors. In addition, according to newly study, antibodies to SARS-CoV-2 have potential for therapeutic passive immunization.[22]

\subsubsection{Monoclonal antibody therapy}

Kohler and Milstein carried out fusion experiments between two known mouse myeloma lines in 1975 and developed a technique for producing antigen-specific MoAbs, that laid the foundation for the possibility of MoAbs to treat tumors.[23] Due to the accessibility to malignant cells in blood and bone marrow, monoclonal antibodies have raised high expectations among scientists for an effective and tolerant treatment of acute myeloid leukemia. Ideal targets represent surface antigens expressed primarily on leukemic blasts while sparing hematopoietic stem cells. In AML, putative targets for antibody targeted therapy include antigens such as CD33 and CD123.[24]

\subsubsection{Anti-CD33 Antibodies}

CD33 is a $67 \mathrm{kD}$ member of the sialic-acid-binding immunoglobulin-like lectins (Siglecs), a discrete subset of the immunoglobulin(Ig) superfamily molecules.[25, 26] As a myeloid differentiation antigen, CD33 primarily expressed at very early stages on myeloid progenitors, also on AML blasts in most patients and some leukemic stem cells.[27, 28] CD33 has been the most commonly exploited target in AML treatment due to its expression on at least one subset of leukemic blasts in almost all the patients.

There are plenty of anti-CD33 antibodies that are under clinical trial and proved effective for therapy of AML, like gemtuzumab ozogamicin(GO), SGN33A (Vadastuximab Talirine)[29], IMGN779 (ImmunoGen) [30], etc. GO will be mainly discussed in this review.

An anti-CD33 antibody, gemtuzumab ozogamicin (GO), have been extensively studied for AML therapy, is the best-known MoAb in AML therapy, also a mixture of success and disappointments.[31] GO is a humanized anti-CD33 MoAb conjugated antibody, carrying a toxic calicheamicin- $\gamma 1$ derivative that, after intracellular hydrolytic release, induces DNA strand breaks, apoptosis, and cell death. GO targets CD33 and rapidly release the toxic part under the acidic condition in the tumor cell lysosomes ( $\mathrm{pH}$ about 4.0) through ahydrolyzable linker.[32, 33]

GO was the first anti-cancer immunoconjugate to obtain regulatory approval from the FDA for older patients with AML in first relapse on the bases of a $30 \%$ overall response rate. [34, 35] However, a decade later, a randomized phase 3 clinical trial was conducted to evaluate the potential benefit of adding GO to standard treatment for induction and post-consolidation in patients with AML, and the addition of GO in the study failed to show any improvement in disease-free survival, CR rates, or overall survival.[36] In 2016, another clinical trial was conducted to compare single-agent GO with best 
supportive care (BSC) including hydroxyurea as first-line therapy in older patients with acute myeloid leukemia unsuitable for intensive chemotherapy. The results showed that first-line monotherapy with low-dose GO significantly improved the overall survival of these patients compared to $\mathrm{BSC}$, and $\mathrm{GO}$ showed undeniable efficacy.[37]

\subsubsection{Anti-CD123 Antibodies}

CD123 is another most common clinically exploited target for MoAb for patients with AML, which promotes increased cell proliferation and survival after combined to interleukin-3 (IL-3R $\alpha$ )[38], as well as relapse and resistance to chemotherapy in leukemia [39].

The widely known anti-CD123 antibodies include JNJ-56022473 (Talacotuzumab, Variant of Former CSL-362)[40], SL-401 (DT388IL3), Anti-KIR(an antibody against killer-cell immunoglobulin receptor (KIR) on NK cells, lirilumab)[41], etc.

One of the most promising anti-CD123 antibodies nowadays is SL-401 (DT388IL3), a recombinant fusion protein composed of the truncated diphtheria toxin and a human IL-3 ligand[42]. SL-401 is internalized after binding to CD123, resulting in inactivation of protein synthesis and cell death. In addition, the drug has received a breakthrough drug designation from the EU and FDA in October 2017 for the treatment of patients with cystic plasmacytoid dendritic cell tumor (BPDCN).[31] A pivotal Phase 2 trial of SL-401 in BPDCN was presented and showed that across all patients and doses, there was an $84 \%$ overall response rate (ORR), and $59 \%$ rate of complete response (CR) indicate great potential of SL-401.[43]

\subsubsection{Chimeric antibody receptor-engineered $T$ cells (CAR-T)}

Chimeric antigen receptor $\mathrm{T}$ cells (also known as CAR $\mathrm{T}$ cells) are $\mathrm{T}$ cells that have been genetically modified to generate artificial $\mathrm{T}$ cell receptors for immunotherapy. Engineered CARs incorporate the specificity of antibody recognition of antigens with the co-stimulatory and activation domains of the $\mathrm{T}$ cell receptor complex.[44] Researchers use genetic engineering to activate $\mathrm{T}$ cells, and add the tumor chimeric antigen receptors (CAR), to make a CAR-T cells. CAR-T cells use "positioning navigation device" CAR, recognize tumor cells specifically, then release a large number of effectors to effectively kill tumor cells, so as to treat malignant tumors.[45]

The use of targeted CAR T cells in patients with some malignant tumors has been shown to lead to sustained disease remission and extended survival.[46-48] A Study of two children with relapsed and refractory ALL have shown that CAR $\mathrm{T}$ cells have the ability to kill even aggressive, treatment-resistant acute leukemia cells in vivo.[49]

\subsubsection{Checkpoint inhibitors}

Immune checkpoints are molecules that are expressed on immune cells and can regulate immune activation. They play an important role in preventing the occurrence of autoimmunity such as immune function abnormalities that attack normal cells. The expression of immune checkpoint molecules on immune cells inhibits the function of immune cells, which prevents the body from generating an efficient anti-tumor immune response and immune escape of tumors happens. Important tumor-related immune checkpoint molecules include: PD1, CTLA4, TIM3, and LAG3, of which PD-1 and CTLA-4 are the most widely studied. ${ }^{[50]}$ Immune checkpoint inhibitors are monoclonal antibodies that are developed to target the particular immune checkpoint. Their main role is to block the interaction between tumor cells that expressing immune checkpoints and immune cells, thus blocking the inhibitory effect of tumor cells on immune cells. Additionally, studies have reported that the high expression of PD-L1, PD-L2, PD-1 and CTLA-4 in AML patients. Thus, blocking immune checkpoints is a potential treatment for AML. CTLA-4 and PD-1 will be mainly discussed in this review.

\subsubsection{CTLA-4}

CTLA-4 is a major T-cell co-stimulatory receptor. After the activation of T cells, CTLA-4 expression was induced to inhibit the $\mathrm{T}$ cell response. ${ }^{[51]}$ This can prevent autoimmune diseases, but it can also inhibit the immune system from killing cancer cells. Therefore, blocking CTLA-4 can improve the efficacy of tumor immunotherapy. Study indicates that T cells treated with a CTLA-4 blocker showed greater cytotoxicity to autologous AML cells than those treated without CTLA-4 blocker, suggesting that CTLA-4 blockade enhances AML-specific T cell function. ${ }^{[52]}$ Findings revealed that using CTLA-4 blockade after hematopoietic stem cell transplantation (HSCT) can establish anti-leukemic effects if autoimmunity can be controlled. ${ }^{[53]}$

\subsubsection{PD-1}

PD-1 (programmed death receptor 1) is also an important immunosuppressive molecule. It modulates the immune system and promotes tolerance by downregulating the response of immune system to human cells and by inhibiting the inflammatory activity of $\mathrm{T}$ cells. ${ }^{[54]}$ Like CTLA-4, PD-1 is a powerful target for immunotherapy, and has effective clinical applications for cancer treatment. There are many ongoing clinical trials that block the immune checkpoint pathway PD1/PD-L1 and allow patients' $T$ cells to fight leukemia cells being evaluated in patients with AML currently. The large number of ongoing clinical trials of AML immunotherapy will improve 
our understanding and enable us to determine the best approach to AML immunotherapy. ${ }^{[5]}$

\section{Discussion}

In the past few decades, few important breakthroughs in the underlying treatment options for AML have occurred. The introduction of immunotherapy has largely changed the landscape of leukemia treatment, offering hope of survival to more leukemia patients. More immunotherapies are being researched and developed, and the field of immunotherapy for cancer will develop more rapidly and diversify in the future. Immunotherapy will likely be the leading treatment option in the future. Many studies have now confirmed breakthroughs in the efficacy of immunotherapy, and we expect that future immunotherapies will be safer and more effective, combining effectively with conventional treatments for the benefit of more cancer patients.

\section{References}

1. KORETH J, SCHLENK R, KOPECKY K J, et al. Allogeneic stem cell transplantation for acute myeloid leukemia in first complete remission: systematic review and meta-analysis of prospective clinical trials [J]. JAMA, 2009, 301(22): 2349-2361.

2. BARRETT A J. Understanding and harnessing the graft-versus-leukaemia effect [J]. Br J Haematol, 2008, 142(6): 877-888.

3. SCHREIBER R D, OLD L J, SMYTH M J. Cancer immunoediting: integrating immunity's roles in cancer suppression and promotion [J]. Science, 2011, 331(6024): 1565-1570.

4. BARRETT A J, LE BLANC K. Immunotherapy prospects for acute myeloid leukaemia [J]. Clin Exp Immunol, 2010, 161(2): 223-232.

5. LION E, WILLEMEN Y, BERNEMAN Z N, et al. Natural killer cell immune escape in acute myeloid leukemia [J]. Leukemia, 2012, 26(9): 2019-2026.

6. STEIN E M, DINARDO C D, POLLYEA D A, et al. Enasidenib in mutant IDH2 relapsed or refractory acute myeloid leukemia [J]. Blood, 2017, 130(6): 722-731.

7. BREEMS D A, VAN PUTTEN W L, HUIJGENS P $\mathrm{C}$, et al. Prognostic index for adult patients with acute myeloid leukemia in first relapse [J]. J Clin Oncol, 2005, 23(9): 1969-1978.

8. STONE R M, MANDREKAR S J, SANFORD B L, et al. Midostaurin plus Chemotherapy for Acute Myeloid Leukemia with a FLT3 Mutation [J]. N Engl J Med, 2017, 377(5): 454-464.

9. DI STASI A, JIMENEZ A M, MINAGAWA K, et al. Review of the Results of WT1 Peptide Vaccination Strategies for Myelodysplastic Syndromes and Acute Myeloid Leukemia from Nine Different Studies [J].
Front Immunol, 2015, 6(36.

10. KEILHOLZ U, LETSCH A, BUSSE A, et al. A clinical and immunologic phase 2 trial of Wilms tumor gene product 1 (WT1) peptide vaccination in patients with AML and MDS [J]. Blood, 2009, 113(26): 6541-6548.

11. PADUA R A, LARGHERO J, ROBIN $\mathrm{M}$, et al. PML-RARA-targeted DNA vaccine induces protective immunity in a mouse model of leukemia [J]. Nat Med, 2003, 9(11): 1413-1417.

12. SUBKLEWE M, GEIGER C, LICHTENEGGER $F$ $\mathrm{S}$, et al. New generation dendritic cell vaccine for immunotherapy of acute myeloid leukemia [J]. Cancer Immunol Immunother, 2014, 63(10): 1093-1103.

13. VAN TENDELOO V F, VAN DE VELDE A, VAN DRIESSCHE A, et al. Induction of complete and molecular remissions in acute myeloid leukemia by Wilms' tumor 1 antigen-targeted dendritic cell vaccination [J]. Proc Natl Acad Sci U S A, 2010, 107(31): 13824-13829.

14. POWLES R, BALCHIN L, FAIRLEY G H, et al. Recognition of leukaemia cells as foreign before and after autoimmunization [J]. Br Med J, 1971, 1(5747): 486-489.

15. MELIEF $\mathrm{C}$ J, VAN DER BURG $\mathrm{S} \quad \mathrm{H}$. Immunotherapy of established (pre)malignant disease by synthetic long peptide vaccines [J]. Nat Rev Cancer, 2008, 8(5): 351-360.

16. GREINER J, DOHNER H, SCHMITT M. Cancer vaccines for patients with acute myeloid leukemia--definition of leukemia-associated antigens and current clinical protocols targeting these antigens [J]. haematologica, 2006, 91(12): 1653-1661.

17. BANCHEREAU J, STEINMAN R M. Dendritic cells and the control of immunity [J]. Nature, 1998, 392(6673): 245-252.

18. NI M, HOFFMANN J M, SCHMITT $M$, et al. Progress of dendritic cell-based cancer vaccines for patients with hematological malignancies [J]. Expert Opin Biol Ther, 2016, 16(9): 1113-1123.

19. DASTMALCHI F, KARACHI A, YANG C, et al. Sarcosine promotes trafficking of dendritic cells and improves efficacy of anti-tumor dendritic cell vaccines via $\mathrm{CXC}$ chemokine family signaling $[\mathrm{J}]$. $\mathrm{J}$ Immunother Cancer, 2019, 7(1): 321.

20. PYZER A R, AVIGAN D E, ROSENBLATT J. Clinical trials of dendritic cell-based cancer vaccines in hematologic malignancies [J]. Human vaccines \& immunotherapeutics, 2014, 10(11): 3125-3131.

21. WALKER L M, BURTON D R. Passive immunotherapy of viral infections: 'super-antibodies' enter the fray [J]. Nat Rev Immunol, 2018, 18(5): 297-308.

22. KLASSE $P$ J, MOORE J P. Antibodies to SARS-CoV-2 and their potential for therapeutic passive immunization [J]. Elife, 2020, 9( 
23. KöHLER G, MILSTEIN C. Continuous cultures of fused cells secreting antibody of predefined specificity [J]. Nature, 1975, 256(5517): 495-497.

24. SAITO Y, KITAMURA H, HIJIKATA A, et al. Identification of Therapeutic Targets for Quiescent, Chemotherapy-Resistant Human Leukemia Stem Cells [J]. Science Translational Medicine, 2010, 2(17): 17ra19-17ra19.

25. CAO H, CROCKER P R. Evolution of CD33-related siglecs: regulating host immune functions and escaping pathogen exploitation? [J]. Immunology, 2011, 132(1): 18-26.

26. CROCKER P R, MCMILLAN S J, RICHARDS H E. CD33-related siglecs as potential modulators of inflammatory responses [J]. 2012, 1253(1): 102-111.

27. HAUSWIRTH A W, FLORIAN S, PRINTZ D, et al. Expression of the target receptor CD33 in CD34+/CD38?/CD123+AML stem cells [J]. European Journal of Clinical Investigation, 2007, 37(1): 73-82.

28. PEIPER S C, ASHMUN R A, LOOK A T. Molecular cloning, expression, and chromosomal localization of a human gene encoding the CD33 myeloid differentiation antigen $[\mathrm{J}] .1988$,

29. KUNG SUTHERLAND M S, WALTER R B, JEFFREY S C, et al. SGN-CD33A: a novel CD33-targeting antibody-drug conjugate using a pyrrolobenzodiazepine dimer is active in models of drug-resistant AML [J]. Blood, 2013, 122(8): 1455-1463.

30. KRYSTAL W M, WALKER R, FISHKIN N, et al. IMGN779, a CD33-targeted antibody-drug conjugate (ADC) with a novel DNA-alkylating effector molecule, induces DNA damage, cell cycle arrest, and apoptosis in AML cells [M]. American Society of Hematology Washington, DC. 2015.

31. MASAROVA L, KANTARJIAN H, RAVANDI F, et al. Update on Immunotherapy in AML and MDS: Monoclonal Antibodies and Checkpoint Inhibitors Paving the Road for Clinical Practice [M]. Springer International Publishing. 2018: 97-116.

32. MAHALLEH M, SHABANI M, RAYZAN E, et al. Reinforcing the primary immunotherapy modulators against acute leukemia; monoclonal antibodies in AML [J]. Immunotherapy, 2019, 11(18): 1583-1600.

33. WALTER R. Antibody-based therapy of acute myeloid leukemia with gemtuzumab ozogamicin [J]. 2013, 18(4): 1311.

34. LARSON R A, SIEVERS E L, STADTMAUER E A, et al. Final report of the efficacy and safety of gemtuzumab ozogamicin (Mylotarg) in patients with CD33-positive acute myeloid leukemia in first recurrence [J]. Cancer, 2005, 104(7): 1442-1452.

35. SIEVERS E L, LARSON R A, STADTMAUER E A, et al. Efficacy and safety of gemtuzumab ozogamicin in patients with CD33-positive acute myeloid leukemia in first relapse [J]. Journal of Clinical Oncology, 2001, 19(13): 3244-3254.
36. PETERSDORF S H, KOPECKY K J, SLOVAK M, et al. A phase 3 study of gemtuzumab ozogamicin during induction and postconsolidation therapy in younger patients with acute myeloid leukemia [J]. Blood, 2013, 121(24): 4854-4860.

37. AMADORI S, SUCIU S, SELLESLAG D, et al. Gemtuzumab Ozogamicin Versus Best Supportive Care in Older Patients With Newly Diagnosed Acute Myeloid Leukemia Unsuitable for Intensive Chemotherapy: Results of the Randomized Phase III EORTC-GIMEMA AML-19 Trial [J]. Journal of Clinical Oncology, 2016, 34(9): 972-979.

38. BAGLEY C J, WOODCOCK J M, STOMSKI F C, et al. The structural and functional basis of cytokine receptor activation: lessons from the common $\beta$ subunit of the granulocyte-macrophage colony-stimulating factor, interleukin-3 (IL-3), and IL-5 receptors $[\mathrm{J}]$. Blood, The Journal of the American Society of Hematology, 1997, 89(5): 1471-1482.

39. TESTA U, RICCIONI R, MILITI S, et al. Elevated expression of IL-3R $\alpha$ in acute myelogenous leukemia is associated with enhanced blast proliferation, increased cellularity, and poor prognosis [J]. Blood, 2002, 100(8): 2980-2988.

40. SMITH B D, ROBOZ G J, WALTER R B, et al. First-in man, phase 1 study of CSL362 (anti-IL3R $\alpha$ /anti-CD123 monoclonal antibody) in patients with $\mathrm{CD} 123+$ acute myeloid leukemia (AML) in CR at high risk for early relapse [M]. American Society of Hematology Washington, DC. 2014.

41. ROMAGNE F, ANDRE P, SPEE P, et al. Preclinical characterization of 1-7F9, a novel human anti-KIR receptor therapeutic antibody that augments natural killer-mediated killing of tumor cells $[\mathrm{J}]$. Blood, 2009, 114(13): 2667-2677.

42. FRANKEL A E, RAMAGE J, KISER M, et al. Characterization of diphtheria fusion proteins targeted to the human interleukin-3 receptor [J]. Protein Engineering, Design and Selection, 2000, 13(8): 575-581.

43. PEMMARAJU N, SWEET K L, LANE A A, et al. Results of pivotal phase 2 trial of SL-401 in patients with blastic plasmacytoid dendritic cell neoplasm (BPDCN) [J]. Blood, 2017, 130(Supplement 1): 1298-1298.

44. JOHN S, CHEN H, DENG M, et al. A Novel Anti-LILRB4 CAR-T Cell for the Treatment of Monocytic AML [J]. Mol Ther, 2018, 26(10): 2487-2495.

45. MAUS M V, JUNE C H. Making Better Chimeric Antigen Receptors for Adoptive T-cell Therapy [J]. Clin Cancer Res, 2016, 22(8): 1875-1884.

46. WANG J, CHEN S, XIAO W, et al. CAR-T cells targeting CLL-1 as an approach to treat acute myeloid leukemia $[\mathrm{J}]$. Journal of Hematology \& Oncology, 2018, 11(1): 
47. PARK J H, RIVIERE I, GONEN M, et al. Long-Term Follow-up of CD19 CAR Therapy in Acute Lymphoblastic Leukemia [J]. N Engl J Med, 2018, 378(5): 449-459.

48. MAUDE S L, LAETSCH T W, BUECHNER J, et al. Tisagenlecleucel in Children and Young Adults with B-Cell Lymphoblastic Leukemia [J]. N Engl J Med, 2018, 378(5): 439-448.

49. GRUPP S A, KALOS M, BARRETT D, et al. Chimeric antigen receptor-modified T cells for acute lymphoid leukemia [J]. N Engl J Med, 2013, 368(16): 1509-1518.

50. YANG H, BUESO-RAMOS C, DINARDO C, et al. Expression of PD-L1, PD-L2, PD-1 and CTLA4 in myelodysplastic syndromes is enhanced by treatment with hypomethylating agents [J]. Leukemia, 2014, 28(6): 1280-1288.

51. LABELLE J L, HANKE C A, BLAZAR B R, et al. Negative effect of CTLA-4 on induction of T-cell immunity in vivo to $\mathrm{B} 7-1+$, but not $\mathrm{B} 7-2+$, murine myelogenous leukemia [J]. Blood, The Journal of the American Society of Hematology, 2002, 99(6): 2146-2153.

52. ZHONG R K, LOKEN M, LANE $\mathrm{T} A$, et al. CTLA-4 blockade by a human MAb enhances the capacity of AML-derived DC to induce T-cell responses against AML cells in an autologous culture system [J]. Cytotherapy, 2006, 8(1): 3-12.

53. FEVERY S, BILLIAU A, SPRANGERS B, et al. CTLA-4 blockade in murine bone marrow chimeras induces a host-derived antileukemic effect without graft-versus-host disease [J]. Leukemia, 2007, 21(7): 1451-1459.

54. OKAZAKI T, CHIKUMA S, IWAI Y, et al. A rheostat for immune responses: the unique properties of PD-1 and their advantages for clinical application [J]. Nature Immunology, 2013, 14(12): 1212-1218.

55. MASAROVA L, KANTARJIAN H, GARCIA-MANNERO $G$, et al. Harnessing the Immune System Against Leukemia: Monoclonal Antibodies and $\mathrm{T}$ 